\title{
Sodium sulfate heptahydrate: a synchrotron energy-dispersive diffraction study of an elusive metastable hydrated salt $\dagger$
}

\author{
Andrea Hamilton* and Christopher Hall \\ Received 30th October 2007, Accepted 2nd January 2008 \\ First published as an Advance Article on the web 30th January 2008 \\ DOI: $10.1039 / b 716734 b$
}

We describe an unusual application of synchrotron energy-dispersive diffraction with hard X-rays to obtain structural information on metastable sodium sulfate heptahydrate. This hydrate was often mentioned in nineteenth and early twentieth century scientific literature but rarely in modern publications, and it had not been characterised structurally. Using a unique three-detector fixed-angle X-ray geometry, a good quality powder diffraction pattern was obtained directly from a stirred suspension of hydrate crystals in saturated aqueous sodium sulfate solution at about $14{ }^{\circ} \mathrm{C}$. The suspension of crystals was contained in the $22 \mathrm{~mm}$ dia sealed cylindrical bottle in which crystallization occurred. Indexing showed that the heptahydrate has a tetragonal unit cell with $a=7.1668 \AA$ and $c=22.2120 \AA$ with a few weak unindexed reflections arising from the $2 a$ supercell. New gravimetric data and the cell dimensions confirm the heptahydrate composition originally proposed by Loewel

(Ann. Chim. Phys., 1850, 29, 62-127).

\section{Introduction}

Sodium sulfate is a salt of great industrial and environmental importance. It is used in the detergent, paper, textile and glass industries, is a by-product of the manufacture of hydrochloric acid and is a major component of natural lacustrine brines and evaporite salt deposits. There is also evidence that it is found on Mars and on Jupiter's satellite Europa. ${ }^{1}$ The decahydrate (Glauber's salt or mirabilite) is the stable phase at temperatures below $32.4{ }^{\circ} \mathrm{C}$, above which it transforms to the anhydrous salt thenardite.

Our interest in sodium sulfate arises from our work on the destructive effects of the crystallization of salts in the pores of stone and concrete. ${ }^{2-10}$ Such salt damage causes considerable harm to buildings and monuments of cultural heritage and more widely in the built environment. Of the commonly occurring salts, sodium sulfate is widely regarded as the most damaging, so that it is frequently used in accelerated durability testing for building stones. ${ }^{11}$ Understanding the damage arising from crystallization pressure depends on being able to predict or to determine analytically which phases crystallize under particular conditions.

Research on sodium sulfate extends back into the nineteenth and early twentieth century when there was much interest in its crystallization behaviour. ${ }^{12-17}$ In particular, there are several detailed accounts which show that mirabilite (sodium sulfate decahydrate) rarely forms directly from supersaturated aqueous solutions, but that the first solid to crystallize is the metastable

Research Laboratory for Conservation Materials Science $\$$, School of Engineering \& Electronics, The University of Edinburgh, Edinburgh, EH93JL,UK.E-mail: andrea.hamilton@ed.ac.uk

$\uparrow$ This paper is part of a JAAS themed issue on Synchrotron Radiation, with guest editors Alex von Bohlen and Metin Tolan.

\$ University of Edinburgh and National Museums Scotland Joint Research Laboratory for Conservation Materials Science. heptahydrate. ${ }^{14-16,18}$ This can be seen as an example of Ostwald's step rule. In the modern literature on stone damage from salts, the heptahydrate is rarely mentioned, and discussion centres almost entirely on mirabilite and thenardite..$^{2,3,5,9,19}$

However, in an important study, Rijniers et al. ${ }^{8}$ were able to estimate dissolved salt concentrations from ${ }^{23} \mathrm{Na}$ NMR measurements during the crystallization of sodium sulfate from supersaturated solutions in porous materials. They found that the dissolved salt concentrations were higher than would be expected if the solution were in equilibrium with mirabilite, but that they corresponded closely to the solubility of the heptahydrate, as given in Gmelin. ${ }^{20}$ We have recently used synchrotron diffraction to show that the first solid phase produced in such porous materials is indeed the heptahydrate [Hamilton and Hall, unpublished]. As part of this study we needed to obtain a high quality reference powder diffraction pattern for the heptahydrate. This paper describes how this was obtained.

The heptahydrate crystallizes readily on cooling a 3.4 molal solution to $10{ }^{\circ} \mathrm{C}$. It shows no strong tendency to transform to mirabilite if kept in a sealed bottle and not cooled much below $0{ }^{\circ} \mathrm{C}$. There is little published information on the heptahydrate-mirabilite transformation. The temperature of conversion to mirabilite is difficult to pinpoint accurately as no systematic study has been carried out. In our experience, the heptahydrate tends to convert promptly to mirabilite if cooled much below $0{ }^{\circ} \mathrm{C}$, if removed from the mother liquor at low temperatures, and occasionally if vigorously shaken at low temperatures. The difficulty in drying, grinding and preserving a sample seriously limits the quality of the data that can be obtained by conventional powder X-ray diffraction. Indeed, we have so far been unable to extract, protect and preserve a sample for laboratory single-crystal structure analysis. Identification of heptahydrate is further complicated by the existence of a so-called heptahydrate pattern in the JCPDS database (card 
no. 40-0727), flagged as being of 'questionable' quality. This card is referenced to a short report by Mehrotra, ${ }^{21}$ which contains no details of sample preparation. We cannot find any relation between the pattern we report here and the set of 21 reflections on the JCPDS card, suggesting what Mehrotra found was not the sodium sulfate heptahydrate which we describe in this paper. Because of its relative instability in air and the need for good sample temperature control, we felt a high quality pattern could only be achieved with powerful in-situ synchrotron methods.

We have therefore made use of synchrotron hard X-ray facilities on station 16.4 at Daresbury Laboratory, UK, to obtain a diffraction pattern from a suspension of crystals in saturated solution in the $22 \mathrm{~mm}$ diameter bottle in which the crystallization was carried out. This avoids all sample transfer and allows the crystals to remain in contact with the saturated mother liquor. To achieve good powder statistics, the suspension of relatively coarse crystals was stirred in the X-ray beam, and an unusually long 60 min data acquisition time was used. Two different methods of stirring were employed. In one, the sample bottle was mounted with its cylinder axis horizontal and rotated about this axis at $1 \mathrm{~Hz}$. The suspension of crystals was continuously carried up the rising inner surface of the bottle to fall back under gravity through the X-ray beam. In the other arrangement, the stationary bottle was mounted vertically and the suspension agitated gently with a magnetic stir-bar. The stir-bar continuously randomized crystal orientation in a rather dense but mobile sedimenting layer with high solids content. This last arrangement was used to acquire the data presented in this paper.

\section{Materials and method}

Heptahydrate samples were prepared from sodium sulfate stock solutions by cooling. Typically, a $3.41 \mathrm{molal}\left(\mathrm{mol} \mathrm{kg}^{-1}\right.$ water) sodium sulfate solution was prepared from AR grade anhydrous sodium sulfate at about $40{ }^{\circ} \mathrm{C} .12 \mathrm{~mL}$ of warm solution was filtered and sealed in a $22 \mathrm{~mm}$ diameter borosilicate bottle. On cooling in an ice bath, heptahydrate crystals usually appear after a few minutes. To confirm that the hydrate produced was the heptahydrate, gravimetric analysis was carried out. This was considered necessary because of the lack of a convincing reference pattern. We have not traced any compositional analysis since the gravimetric work reported by Loewel ${ }^{15,16}$ in $1850-51$. Indeed, Loewel's first results suggested to him that the hydrate he formed was in fact the octahydrate. ${ }^{15}$ Later refinement of the experimental method, which involved crystallization from water-ethanol solutions, produced results from which he concluded that it was the heptahydrate. ${ }^{16}$ We carried out our own gravimetric experiments, measuring the mass loss on heating heptahydrate samples in an air oven at $140-160{ }^{\circ} \mathrm{C}$ to constant mass. This apparently simple procedure is far from straightforward because of the difficulties involved in extracting crystals of the metastable hydrate from the mother liquor and drying them. However, in a series of replicate analyses we obtained the composition $\mathrm{Na}_{2} \mathrm{SO}_{4} \cdot n \mathrm{H}_{2} \mathrm{O}, n=7.4 \pm 0.4$ over 10 samples, which is close to Loewel's figure of $n=7.3 \pm 0.3$ averaged over 13 samples. It is probable that the small positive deviation from 7 arises from incomplete drying of the crystals and/or solution inclusions in the crystals.

\section{Energy-dispersive synchrotron diffraction}

The penetration of X-rays through solids and liquids increases rapidly with increasing X-ray photon energy. X-rays from a conventional $\mathrm{Cu} \mathrm{K} \alpha$ laboratory X-ray tube (with photon energy $7.5 \mathrm{keV}$ ) are strongly attenuated over distances of order $1 \mathrm{~mm}$, so that thin-walled capillary tubes or flat plate mounts are normally used. However, by raising the photon energy into the range 20-90 $\mathrm{keV}$, we can achieve much greater "stopping distances" (1/e decay lengths), for example, for water, glass and minerals of the lighter elements of at least 10-20 $\mathrm{mm}$. Synchrotron sources can provide hard X-rays with such energies, and higher. In the work we describe here, we have used polychromatic ("white beam") $\mathrm{X}$-rays with energies in the range 20 to $90 \mathrm{keV}$ to carry out a diffraction experiment in energy-dispersive mode. ${ }^{22}$ The optics arrangement is particularly simple, as shown in Fig. 1, since we collect Bragg scattered $\mathrm{X}$-rays at a fixed diffraction angle $2 \theta$ using a parallel plate collimator and an energy dispersive Ge detector. From the Bragg equation, $E d \sin \theta=6.1992$ (where $E$ is the $\mathrm{X}$-ray photon energy in $\mathrm{keV}$ and $d$ the $d$-spacing in $\AA$ ) we derive a powder pattern equivalent to that collected in a conventional monochromatic angle-dispersive experiment. The energy-dispersive diffractometer used is unique in having three fixed-angle detectors which collect diffracted X-rays from the sample simultaneously. ${ }^{23}$ Each detector is set at a different angle, and the combined dataset gives a wide coverage of $d$-spacings, roughly from $6.7-1.3 \AA$. The combination of high penetration and fixed X-ray optics makes energy dispersive diffraction highly suitable for in-situ measurements on large volume sample cells. In the present case, we collect diffracted X-rays in transmission through a glass bottle of $22 \mathrm{~mm}$ diameter containing a suspension of heptahydrate crystals in saturated sodium sulfate mother liquor. The sample bottle was in contact with an aluminium block maintained at a temperature controlled by circulation of a glycol-water mixture giving a sample temperature of $\mathrm{ca} .14^{\circ} \mathrm{C}$.

As the sample contains suspended solid particles with a considerable amount of free water, the XRD pattern shows a large,

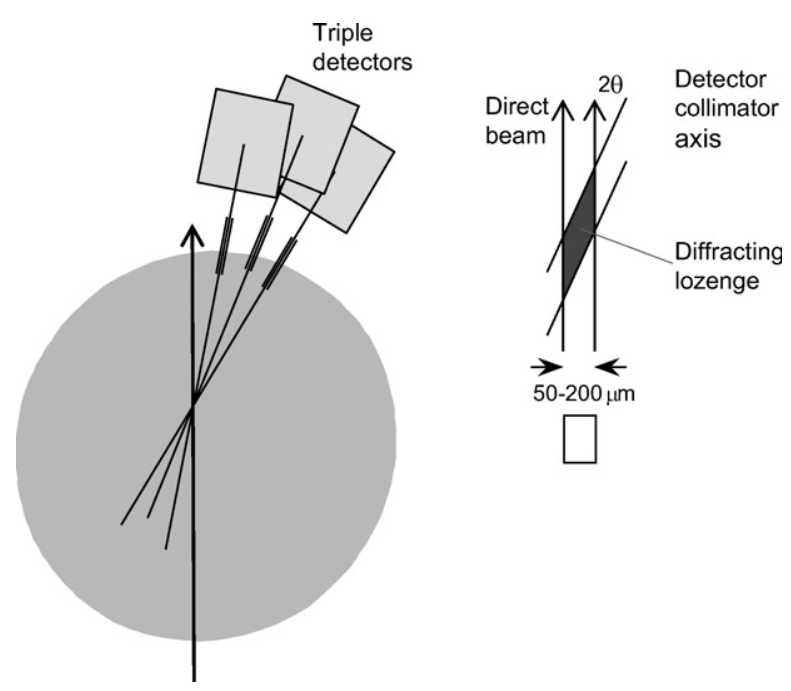

Fig. 1 Schematic diagram of the detector system used on station 16.4 at Daresbury Laboratory, UK. Each collimated detector captures diffracted $\mathrm{X}$-rays at a fixed scattering angle from a small "lozenge" in the sample. 


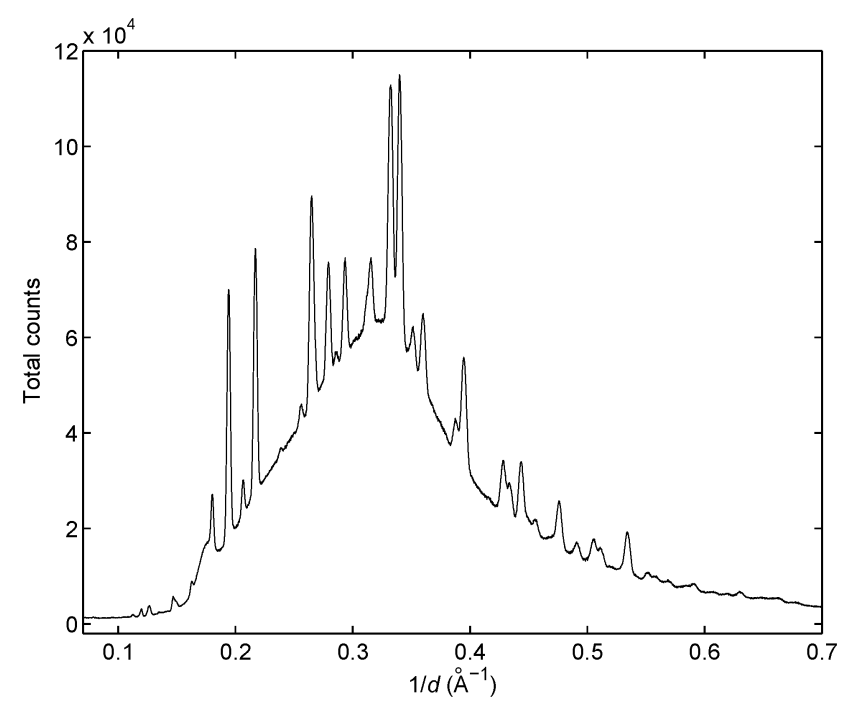

Fig. 2 Raw data from the middle detector only at a sample temperature of approx. $14{ }^{\circ} \mathrm{C}$. Diffraction pattern shows heptahydrate diffraction peaks superimposed on a large diffuse background from the liquid water in which the crystals are suspended.

diffuse scattering background (see Fig. 2). Raw data were processed by removing the diffuse background using a spline interpolation between 20 intensity values at manually selected channels chosen to lie well away from the diffraction peaks; and by merging the background-corrected patterns from the three detectors a composite powder pattern was obtained. The resulting pattern after processing is shown as Fig. 3 .

Accurate peak positions were required for indexing, which was achieved within the experimental limits of the detector system by Gaussian peak fitting. While energy-dispersive diffraction characteristically gives rather broad diffraction peaks (here FWHM about $0.0035 \AA^{-1}$ ) with peak widths set by the detector resolution

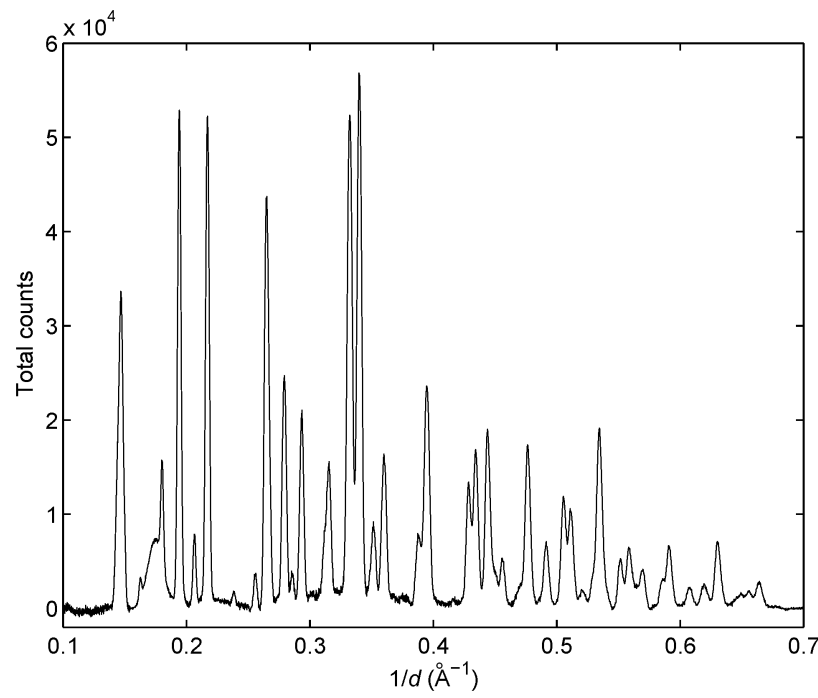

Fig. 3 Energy dispersive powder diffraction pattern from slurry of metastable hydrate in water at approx $14{ }^{\circ} \mathrm{C}$. Diffuse background has been removed and data from three fixed angle detectors merged. Detector angles are $2.007^{\circ}, 4.875^{\circ}$ and $7.735^{\circ} 2 \theta$. The pattern is obtained by summing 120 data scans each of $30 \mathrm{~s}$ duration. (typically about $0.3 \mathrm{keV}$ ), the peaks are usually well represented by Gaussians. ${ }^{24}$ As a result, the $d$-spacings may be found with surprising precision. We determined a set of $d$-spacings along with relative peak intensities by manual fitting (and in a few cases by deconvolving overlapping peaks), and these $d$-spacings were the basis for indexing the pattern.

\section{Indexing}

The indexing programs Treor90 and McMaille were used, and the parameters refined using Eracel. The unit cell is tetragonal, with cell parameters $a=7.1668 \AA$ and $c=22.2120 \AA$, cell volume $1140.9 \AA^{3}$ Gans $^{25}$ gives an experimental crystal density $1.53 \pm$ $0.02 \mathrm{~g} \mathrm{~cm}^{-3}$, so that $Z=3.92 \pm 0.05$ based on the chemical formula $\mathrm{Na}_{2} \mathrm{SO}_{4} \cdot 7 \mathrm{H}_{2} \mathrm{O}$, which is satisfactorily close to 4 . The crystallographic density based on the cell volume and the heptahydrate composition is $1.561 \mathrm{~g} \mathrm{~cm}^{-3}$. We get de Wolff figure-ofmerit $\mathrm{M}$ values of 15-22. Systematic absences are consistent with the space group $I 4_{1} / a$. The observed and calculated peak positions are shown in Table 1. In achieving this indexing, seven peaks were omitted. These seven unindexed peaks (most of low intensity) appear to be diffraction lines and were seen in several earlier datasets. They are seen simultaneously in two energydispersive detectors, strong evidence that they are indeed diffraction lines rather than escape peaks or fluorescence lines. The solution from which the heptahydrate was prepared was scrupulously filtered, so they are unlikely to be from impurities. All seven lines are in fact satisfactorily identified as weak $2 a, 2 b$ supercell reflections, as shown in Table 1.

We note the interesting recent report by Genkinger and Putnis $^{26}$ on a crystalline sodium sulfate hydrate which these authors call selmaite. All but one of the 14 selmaite diffraction peaks correspond to strong peaks in our heptahydrate pattern. We conclude that selmaite is the same substance as heptahydrate, although the selmaite $d$-spacings are displaced to lower $2 \theta$ by $0.20^{\circ}$ compared with our $d$-spacings. This zero-point error is presumably the result of sample misalignment. The monoclinic unit cell tentatively proposed by Genkinger and Putnis is different from that which we report here, and in our view not correct. Interestingly, Genkinger and Putnis match the Mehrotra powder pattern to a hydrate formed during the rehydration of thenardite, consequently misidentifying this as the heptahydrate. This suggests the possible existence of another hydrate of sodium sulfate. Further investigation of this is now under way.

\section{Comments and conclusions}

Our need for a reliable powder pattern arose from our work on the crystallization of sodium sulfate solutions in porous materials. We have shown that a reproducible powder pattern for the heptahydrate, distinctly different from the JCPDS reference card, can be best achieved with in-situ synchrotron methods. We have also recently observed the crystallization of the heptahydrate within the pores of several porous materials by using hard X-ray methods similar to those described here. This suggests that the formation of heptahydrate should be incorporated in physical theories of salt crystallization damage, which currently is not the case.

Work is in progress to obtain a full crystal structure. 
Table 1 Observed diffraction lines for sodium sulfate heptahydrate, and indexed lines predicted from tetragonal unit cell with $a=7.1668 \AA$ and $c=$ $22.2120 \AA$

\begin{tabular}{|c|c|c|c|c|c|c|c|c|}
\hline$d / \AA ̊$ obs & $\begin{array}{l}d / \AA ̊ \text { obs with zero } \\
\text { correction }^{a}\end{array}$ & $d / \AA ̊$ calc & ${ }^{\circ} 2 \theta \mathrm{calc}^{b}$ & $h^{\mathrm{c}}$ & $k^{\mathrm{c}}$ & $l^{c}$ & $I$ & Comments \\
\hline 6.8176 & 6.8253 & 6.8205 & 12.970 & 1 & 0 & 1 & 35 & \\
\hline 5.5510 & 5.5561 & 5.5530 & 15.946 & 0 & 0 & 4 & 16 & \\
\hline 5.1499 & 5.1544 & 5.1495 & 17.206 & 1 & 0 & 3 & 55 & \\
\hline 4.8464 & 4.8502 & 4.8462 & 18.292 & 2 & 1 & 3 & 8 & 2a supercell \\
\hline 4.6089 & 4.6124 & 4.6104 & 19.236 & 1 & 1 & 2 & 55 & \\
\hline 3.7734 & 3.7757 & 3.7758 & 23.543 & 1 & 0 & 5 & 45 & \\
\hline 3.5815 & 3.5836 & 3.5834 & 24.827 & 2 & 0 & 0 & 25 & \\
\hline 3.5016 & 3.5032 & 3.5025 & 25.410 & 3 & 2 & 3 & 2 & 2a supercell \\
\hline 3.4088 & 3.4107 & 3.4103 & 26.109 & 2 & 0 & 2 & 20 & \\
\hline 3.2082 & 3.2098 & 3.2051 & 27.813 & 4 & 2 & 0 & 3 & 2a supercell \\
\hline 3.1708 & 3.1725 & 3.1722 & 28.107 & 2 & 1 & 1 & 15 & \\
\hline 3.0098 & 3.0113 & 3.0109 & 29.646 & 2 & 0 & 4 & 52 & \\
\hline 2.5324 & 2.5334 & 2.5338 & 35.397 & 2 & 2 & 0 & 25 & \\
\hline 2.3338 & 2.3347 & 2.3335 & 38.550 & 1 & 0 & 9 & 15 & \\
\hline 2.3024 & 2.3032 & 2.3052 & 39.043 & 2 & 2 & 4 & 20 & \\
\hline 2.2522 & 2.2530 & 2.2550 & 39.949 & 2 & 1 & 7 & 22 & \\
\hline 2.1921 & 2.1928 & 2.1948 & 41.093 & 2 & 0 & 8 & 5 & \\
\hline 2.0985 & 2.0992 & 2.0983 & 43.074 & 3 & 1 & 4 & 18 & \\
\hline 2.0332 & 2.0339 & 2.0344 & 44.499 & 1 & 1 & 10 & 7 & \\
\hline 1.9783 & 1.9789 & 1.9798 & 45.795 & 3 & 2 & 1 & 12 & \\
\hline 1.9573 & 1.9579 & 1.9554 & 46.398 & 2 & 1 & 9 & 10 & \\
\hline 1.9202 & 1.9208 & 1.9197 & 47.313 & 3 & 2 & 3 & 1 & \\
\hline 1.8699 & 1.8704 & 1.8716 & 48.607 & 2 & 2 & 8 & 20 & \\
\hline 1.8128 & 1.8133 & 1.8144 & 50.245 & 3 & 2 & 5 & 2 & \\
\hline 1.7895 & 1.7900 & 1.7917 & 50.926 & 4 & 0 & 0 & 7 & \\
\hline 1.7560 & 1.7565 & 1.7557 & 52.047 & 3 & 1 & 8 & 4 & \\
\hline 1.3640 & 1.3642 & 1.3641 & 68.761 & 5 & 0 & 5 & 2 & \\
\hline 1.3452 & 1.3454 & 1.3446 & 69.905 & 3 & 3 & 10 & 2 & \\
\hline 1.2965 & 1.2967 & 1.2957 & 72.954 & 3 & 2 & 13 & 1 & \\
\hline
\end{tabular}

${ }^{a}$ Zero point error $-0.015 \AA^{-1}$ in $1 / d .{ }^{b}$ Equivalent $2 \theta$ calculated for $\lambda=1.54056 \AA\left(\mathrm{Cu} \mathrm{K} \alpha_{1}\right){ }^{c}$ For supercell reflections, $h$ and $k$ are referred to the $2 a, 2 b$ cell parameters of the $2 a, 2 b, c$ supercell.

\section{Acknowledgements}

We thank EPSRC for financial support; Leo Pel and Kristina Terheiden for collaboration on heptahydrate crystallization; Jeremy Cockcroft for help with indexing; Colin Pulham for discussions and advice; Alfie Neild and David Taylor for support with synchrotron instrumentation.

\section{References}

1 W. M. Grundy, B. J. Buratti, A. F. Cheng, J. P. Emery, A. Lunsford, W. B. McKinnon, J. M. Moore, S. F. Newman, C. B. Olkin, D. C. Reuter, P. M. Schenk, J. R. Spencer, S. A. Stern, H. B. Throop and H. A. Weaver, Science, 2007, 318, 234-237.

2 R. J. Flatt, J. Cryst. Growth, 2002, 242, 435-454.
3 C. Rodriguez-Navarro, E. Doehne and E. Sebastian, Cem. Concr. Res., 2000, 30, 1527-1534.

4 G. W. Scherer, Cem. Concr. Res., 1999, 29, 1347-1358.

5 N. Tsui, R. J. Flatt and G. W. Scherer, J. Cultural Heritage, 2003, 4, $109-115$.

6 C. W. Correns, Discuss. Faraday Soc., 1949, 5, 267-271.

7 R. J. Flatt, M. Steiger and G. W. Scherer, Environ. Geol., 2007, 52, 187-203.

8 L. A. Rijniers, H. P. Huinink, L. Pel and K. Kopinga, Phys. Rev. Lett., 2005, 94, Art 07553.

9 M. Steiger, J. Cryst. Growth, 2005, 282, 455-469.

10 M. Steiger, J. Cryst. Growth, 2005, 282, 470-481.

11 C. A. Price, Archit. J., 1975, 2, 337-339.

12 C. Balarew, Z. Anorg. Allg. Chem., 1965, 336, 92-95.

13 R. D. Eddy and A. W. C. Menzies, J. Phys. Chem., 1940, 44, 207-235.

14 H. Hartley, B. M. Jones and G. A. Hutchinson, J. Chem. Soc. Trans., $1908,93,825-833$.

15 H. Loewel, Ann. Chim. Phys., 1850, 29, 62-127. 
16 H. Loewel, Ann. Chim. Phys., 1851, 33, 334-337.

17 E. R. Washburn and W. J. Clem, J. Am. Chem. Soc., 1938, 60, 754 757.

18 L. C. de Coppet, Bull. Soc. Vaudoise Sci. Nat., 1901, 37, 455-462.

19 C. Rodriguez-Navarro and E. Doehne, Earth Surf. Processes Landforms, 1999, 24, 191.

20 L. Gmelin, Handbuch der Anorganischen Chemie, Springer-Verlag, Berlin, vol. 21, 1966.
21 B. N. Mehrotra, Acta Crystallogr., 1987, A43, C119.

22 D. Häusermann and P. Barnes, Phase Transitions, 1992, 39, 99-115.

23 P. Barnes, A. C. Jupe, S. L. Colston, S. D. Jacques, A. Grant,

T. Rathbone, M. Miller, S. M. Clark and R. J. Cernik, Nucl. Instrum. Methods Phys. Res., Sect. B, 1998, 134, 310-313.

24 J. W. Otto, J. Appl. Crystallogr., 1997, 30, 1008-1015.

25 W. Gans, Z. Phys. Chem., 1978, 111, 39-46.

26 S. Genkinger and A. Putnis, Environ. Geol., 2007, 52, 329-337. 